\title{
Characterization of Closed Head Impact Injury in Rat
}

\author{
Yi Hua, ${ }^{1}$ Praveen Akula, ${ }^{1}$ Matthew Kelso, ${ }^{2}$ and Linxia Gu ${ }^{1}$ \\ ${ }^{1}$ Department of Mechanical and Materials Engineering, University of Nebraska-Lincoln, Lincoln, NE 68588-0656, USA \\ ${ }^{2}$ Department of Pharmacy Practice, University of Nebraska Medical Center, Omaha, NE 68198-6045, USA \\ Correspondence should be addressed to Linxia Gu; lgu@unl.edu
}

Received 7 December 2014; Accepted 10 March 2015

Academic Editor: Mauro S. Oliveira

Copyright $\odot 2015$ Yi Hua et al. This is an open access article distributed under the Creative Commons Attribution License, which permits unrestricted use, distribution, and reproduction in any medium, provided the original work is properly cited.

\begin{abstract}
The closed head impact (CHI) rat models are commonly used for studying the traumatic brain injury. The impact parameters vary considerably among different laboratories, making the comparison of research findings difficult. In this work, numerical $\mathrm{CHI}$ experiments were conducted to investigate the sensitivities of intracranial responses to various impact parameters (e.g., impact depth, velocity, and position; impactor diameter, material, and shape). A three-dimensional finite element rat head model with anatomical details was subjected to impact loadings. Results revealed that impact depth and impactor shape were the two leading factors affecting intracranial responses. The influence of impactor diameter was region-specific and an increase in impactor diameter could substantially increase tissue strains in the region which located directly beneath the impactor. The lateral impact could induce higher strains in the brain than the central impact. An indentation depth instead of impact depth would be appropriate to characterize the influence of a large deformed rubber impactor. The experimentally observed velocity-dependent injury severity could be attributed to the "overshoot" phenomenon. This work could be used to better design or compare CHI experiments.
\end{abstract}

\section{Introduction}

Traumatic brain injury (TBI) is the leading cause of mortality and morbidity in the United States, which affects over 1.7 million Americans each year [1]. It leads to long-term disability in cognition, sensorimotor function, and personality [2]. To study the mechanisms of TBI, the rat closed head impact (CHI) is commonly used for replicating the trauma events [39]. In a typical CHI procedure, the rat is fully anesthetized and secured in a stereotactic frame. An impact load is delivered directly to the intact skull through a pneumatically driven impactor. Compared with other animal models such as fluid percussion [10] and controlled cortical impact (CCI) [11], the CHI model avoided performing the craniotomy and thus the risk of operation-induced inflammation. The $\mathrm{CHI}$ model has been used for investigating the cortical tissue loss $[3,4]$, acute subarachnoid hemorrhage [5], diffuse axonal injury [6], blood-brain barrier dysfunction [7,8], and concussion [9]. Nevertheless, various control parameters (e.g., impact depth, velocity, and position; impactor diameter, material, and shape) were employed by different research groups, making it hard to compare these results. In addition, the internal brain response variables could be documented in more detail. To address these shortcomings, it is crucial to establish the linkage between external impact parameters and intracranial responses.

In this work, the sensitivities of intracranial responses to various impact parameters in the $\mathrm{CHI}$ model were systematically investigated. A three-dimensional (3D) finite element (FE) rat head model with anatomical details was developed from medical images. An orthogonal experimental design was implemented for carrying out eight computational experiments to correlate the regional brain mechanics with impact controls including impact depth, velocity, and position, as well as impactor diameter, material, and shape.

\section{Finite Element Modeling}

A 3D FE rat head model was generated from the high-resolution magnetic resonance imaging (MRI) datasets of an adult male Sprague-Dawley rat weighing about $360 \mathrm{~g}$, as shown in Figure 1. The brain MRI has an isotropic resolution of 
TABLE 1: Material properties of the rat head components [13-17].

(a) Elastic material properties

\begin{tabular}{lccc}
\hline Component & Density $\left(\mathrm{kg} / \mathrm{m}^{3}\right)$ & Young's modulus $(\mathrm{MPa})$ & Poisson's ratio $(/)$ \\
\hline Skull & 1710 & 6000 & 0.3 \\
\hline
\end{tabular}

(b) Viscoelastic material properties

\begin{tabular}{lcccc}
\hline Component & Density $\left(\mathrm{kg} / \mathrm{m}^{3}\right)$ & Short-term shear modulus $(\mathrm{kPa})$ & Long-term shear modulus $(\mathrm{kPa})$ & Decay constant $(\mathrm{ms})$ \\
\hline Cerebrum & 1040 & 1.72 & 0.51 & 20 \\
Cerebellum & 1040 & 1.20 & 0.36 & 20 \\
Hippocampus & 1040 & 4.06 & 0.61 & 20 \\
\hline
\end{tabular}

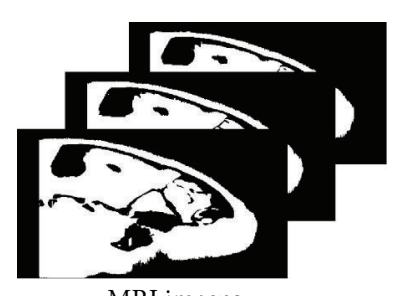

MRI images

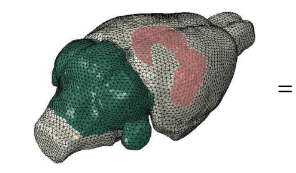

Rat brain assembly
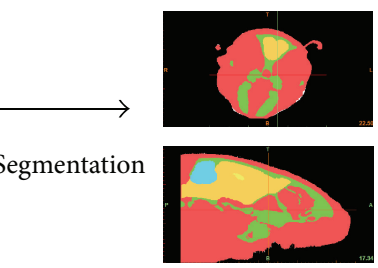

Segmented rat head
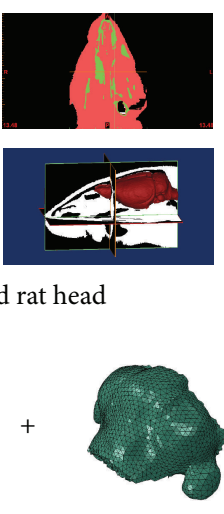

Cerebellum

FIGURE 1: Finite element discretization of the adult male Sprague-Dawley rat head.

$256 \times 256 \times 256$ pixels, for a field view of $30 \mathrm{~mm}$ in all three directions. The image data were segmented into three main brain regions: cerebrum, hippocampus, and cerebellum. The segmentation was realized using the 3D image analysis algorithm implemented in Mimics (Materialise, Inc., Leuven, Belgium). The segmented brain model was then imported into HyperMesh (Altair Engineering, Inc., MI, USA) and meshed as a triangular surface mesh (S3R). A volume mesh with 4-noded tetrahedral elements (C3D4) was generated from this surface mesh. The rat skull was created by offsetting a layer of wedge elements (C3D6) above the outer surface of the brain by $0.16 \mathrm{~mm}$ [12]. A mesh convergence test was conducted and the minimum mesh size of $0.4 \mathrm{~mm}$ was chosen. At this resolution, the rat head model consisted of a total of 1,107,183 tetrahedral elements and 14,898 prism elements for the brain and skull, respectively.

The rat brain was assumed to be a linear viscoelastic material with a decay constant of $20 \mathrm{~ms}$ [13]. For the cerebrum, a short-term shear modulus of $1.72 \mathrm{kPa}$ and a long-term shear modulus of $0.51 \mathrm{kPa}$ were assumed. This assumption was based on the indentation test results obtained from the adult rat brain as reported by Gefen et al. [14]. The cerebellum had a short-term modulus of $1.2 \mathrm{kPa}$ and a long-term modulus of $0.36 \mathrm{kPa}$ [15], while the same parameters for the hippocampus were $4.06 \mathrm{kPa}$ and $0.61 \mathrm{kPa}$, respectively [16]. The rat skull was modeled as a homogeneous linear elastic isotropic material and Young's modulus and Poisson's ratio were assumed as $6 \mathrm{GPa}$ and 0.3 , respectively [17]. A summarization of the material properties is described in Table 1.

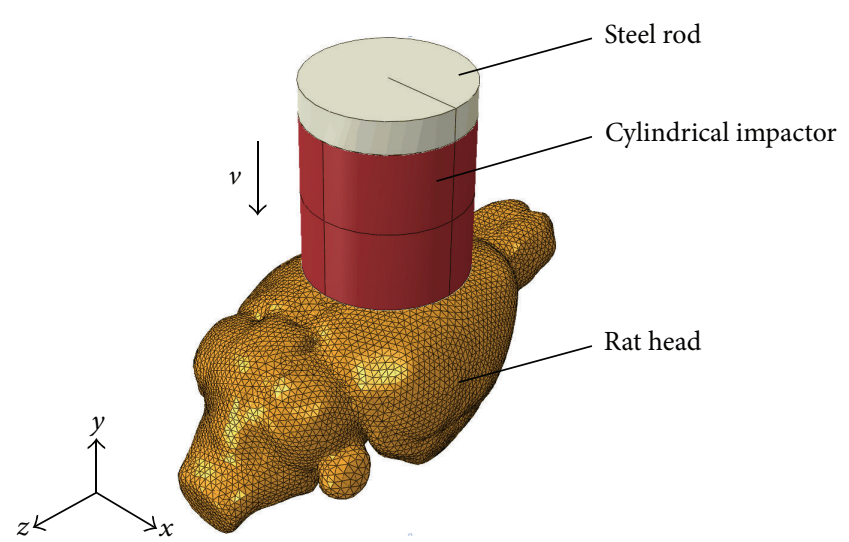

Figure 2: Isometric view of the closed head impact model.

To replicate the experimental CHI procedure, a cylindrical impactor, which connected to the bottom surface of a steel rod, was positioned perpendicular to the dorsal surface of the rat skull (Figure 2). A linearly ramping displacement was enforced onto the steel rod to achieve the prescribed impact depth and velocity. Due to variations in impactor geometries, materials, and positions, the prescribed conditions will result in different indentation behaviors. The interactions between the impactor and skull as well as between the brain and skull were modeled through penalty contact algorithm with tangential sliding and hard contact normal behavior. The nodes on the bottom surface of the skull were constrained in 
TABLE 2: Assignment of six factors and their selected levels in the $\mathrm{L}_{8}\left(2^{7}\right)$ orthogonal array and predicted peak maximum principal strain (MPS) in the cerebrum, hippocampus, and cerebellum.

\begin{tabular}{|c|c|c|c|c|c|c|c|c|c|}
\hline \multirow{2}{*}{ Case number } & \multicolumn{6}{|c|}{ Factors } & \multicolumn{3}{|c|}{ Predicted peak MPS } \\
\hline & $\mathrm{A}$ & $\mathrm{B}$ & $\mathrm{C}$ & $\mathrm{D}$ & $\mathrm{E}$ & $\mathrm{F}$ & Cerebrum & Hippocampus & Cerebellum \\
\hline 1 & 1 & 3 & Central & 6 & Steel & Flat & 0.1775 & 0.1466 & 0.1535 \\
\hline 2 & 1 & 3 & Lateral & 6 & Nylon & Convex & 0.2283 & 0.1593 & 0.0326 \\
\hline 3 & 1 & 6 & Central & 12 & Steel & Convex & 0.2061 & 0.0912 & 0.0494 \\
\hline 4 & 1 & 6 & Lateral & 12 & Nylon & Flat & 0.4080 & 0.2993 & 0.1261 \\
\hline 5 & 2 & 3 & Central & 12 & Nylon & Flat & 0.6192 & 0.3644 & 0.3890 \\
\hline 6 & 2 & 3 & Lateral & 12 & Steel & Convex & 0.3744 & 0.3080 & 0.1238 \\
\hline 7 & 2 & 6 & Central & 6 & Nylon & Convex & 0.3047 & 0.2204 & 0.1976 \\
\hline 8 & 2 & 6 & Lateral & 6 & Steel & Flat & 0.4982 & 0.4092 & 0.2893 \\
\hline
\end{tabular}

A (impact depth, $\mathrm{mm}$ ), B (impact velocity, $\mathrm{m} / \mathrm{s}$ ), C (impact position), D (impactor diameter, $\mathrm{mm}$ ), $\mathrm{E}$ (impactor material), and $\mathrm{F}$ (impactor shape).

all six degrees of freedom to avoid rigid body translation. The FE model was solved using the nonlinear transient dynamic procedure Abaqus/Explicit (Dassault Systems Simulia Corp., RI, USA).

\section{Design of Computational Experiments}

A six-factor two-level orthogonal experimental design was implemented to systematically investigate the sensitivities of intracranial responses to various impact parameters in three different brain regions: cerebrum, hippocampus, and cerebellum. The six factors studied were the prescribed impact depth (A), velocity (B), and position (C), as well as impactor diameter (D), material (E), and shape (F). The baseline level of these factors was selected from the typical $\mathrm{CHI}$ procedures, including an impact depth of $1 \mathrm{~mm}$, velocity of $3 \mathrm{~m} / \mathrm{s}$, central position around the midline between bregma and lambda, nylon impactor with a diameter of $6 \mathrm{~mm}$, and a flat end. The second level of the quantitative factors, that is, impact depth, velocity, and impactor diameter, was double over the baseline. For the qualitative factors, the second level was selected as lateral impact position over the left parietal bone between bregma and lambda, steel impactor material, and convex end. $\mathrm{An}_{8}(2)^{7}$ orthogonal array from the module of Statistica (Version 10.0) was adopted to implement the multifactor combination. The assignment of six factors and their selected levels in the array was depicted in Table 2.

\section{Results}

4.1. Model Verification. The published CCI injury data [13] were used to verify the FE model. To simulate the cortical impact, a $7 \mathrm{~mm}$ diameter craniotomy was created on the left skull. The impactor shape and impact direction were accurately defined according to the settings in the cited publication. The relative position between the impactor and brain is shown in Figure 3(a). The impact depth and velocity were assumed to be $1.5 \mathrm{~mm}$ and $4 \mathrm{~m} / \mathrm{s}$, respectively. The peak maximum principal strain (MPS) was extracted at four different locations of the brain (Figure 3(a), locations $1-4)$, corresponding to the superior cortex, deep cortex, hippocampus, and thalamus as measured in [13].
TABLE 3: Range analysis for the peak maximum principal strain (MPS) in the cerebrum, hippocampus, and cerebellum.

\begin{tabular}{lcccccc}
\hline Factors & $\mathrm{A}$ & $\mathrm{B}$ & $\mathrm{C}$ & $\mathrm{D}$ & $\mathrm{E}$ & $\mathrm{F}$ \\
\hline $\begin{array}{l}\text { Cerebrum } \\
K_{1}\end{array}$ & 0.2550 & 0.3499 & 0.3269 & 0.3022 & 0.3141 & 0.4257 \\
$K_{2}$ & 0.4491 & 0.3543 & 0.3772 & 0.4019 & 0.3901 & 0.2784 \\
$R$ & 0.1941 & 0.0044 & 0.0503 & 0.0997 & 0.0760 & 0.1473 \\
\hline Hippocampus & & & & & & \\
$K_{1}$ & 0.1741 & 0.2446 & 0.2057 & 0.2339 & 0.2388 & 0.3049 \\
$K_{2}$ & 0.3255 & 0.2550 & 0.2940 & 0.2657 & 0.2609 & 0.1947 \\
$R$ & 0.1514 & 0.0104 & 0.0883 & 0.0318 & 0.0221 & 0.1102 \\
\hline Cerebellum & & & & & & \\
$K_{1}$ & 0.0904 & 0.1747 & 0.1974 & 0.1683 & 0.1540 & 0.2395 \\
$K_{2}$ & 0.2499 & 0.1656 & 0.1430 & 0.1721 & 0.1863 & 0.1009 \\
$R$ & 0.1595 & 0.0091 & 0.0544 & 0.0038 & 0.0323 & 0.1386 \\
\hline
\end{tabular}

A (impact depth), B (impact velocity), C (impact position), D (impactor diameter), $\mathrm{E}$ (impactor material), and $\mathrm{F}$ (impactor shape).

Comparative results are shown in Figure 3(b). The peak MPS predicted by the FE model agreed well with the published data. The maximum deviation between the FE model and [13] was $23.8 \%$ at location 4 , while the deviations at other locations were less than $9.0 \%$. Moreover, in the FE model, location 2 experienced 13.0\% higher peak MPS than location 1 although location 1 was more close to the impact site. This was consistent with the finding in [13], which measured a $17.4 \%$ higher peak MPS at deep cortex compared to the superior cortex.

4.2. Sensitivity Studies. The predicted MPS was used to characterize the brain responses. The peak MPS in the cerebrum, hippocampus, and cerebellum for all eight cases are listed in Table 2. The range analysis, which assumes that the influence of other factors on the result is balanced when analyzing the impact of a specific factor, was performed to quantify the significance level of each factor as shown in Table 3. The $K_{i}$ value of a factor was the average of four values of peak MPS for level $i$ listed in Table 2, and the range value $R$ for each factor was the difference between $K_{i}$ values of the two levels. 


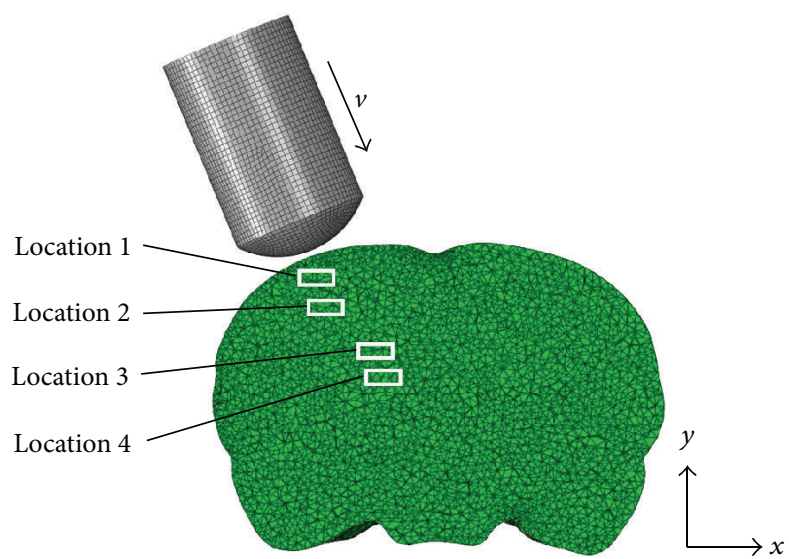

(a)

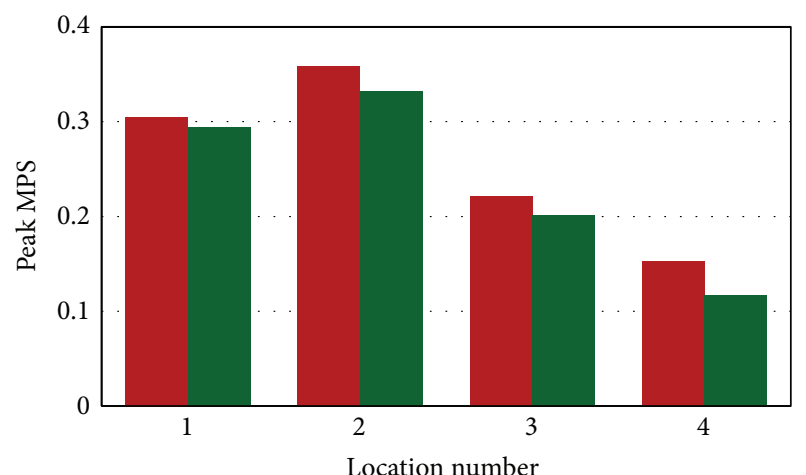

Ref. [13]

FE model

FIGURE 3: Verification of rat head model with [13]. (a) Coronal view of rat head subjected to controlled cortical impact and (b) peak MPS comparisons at four different locations of the brain.
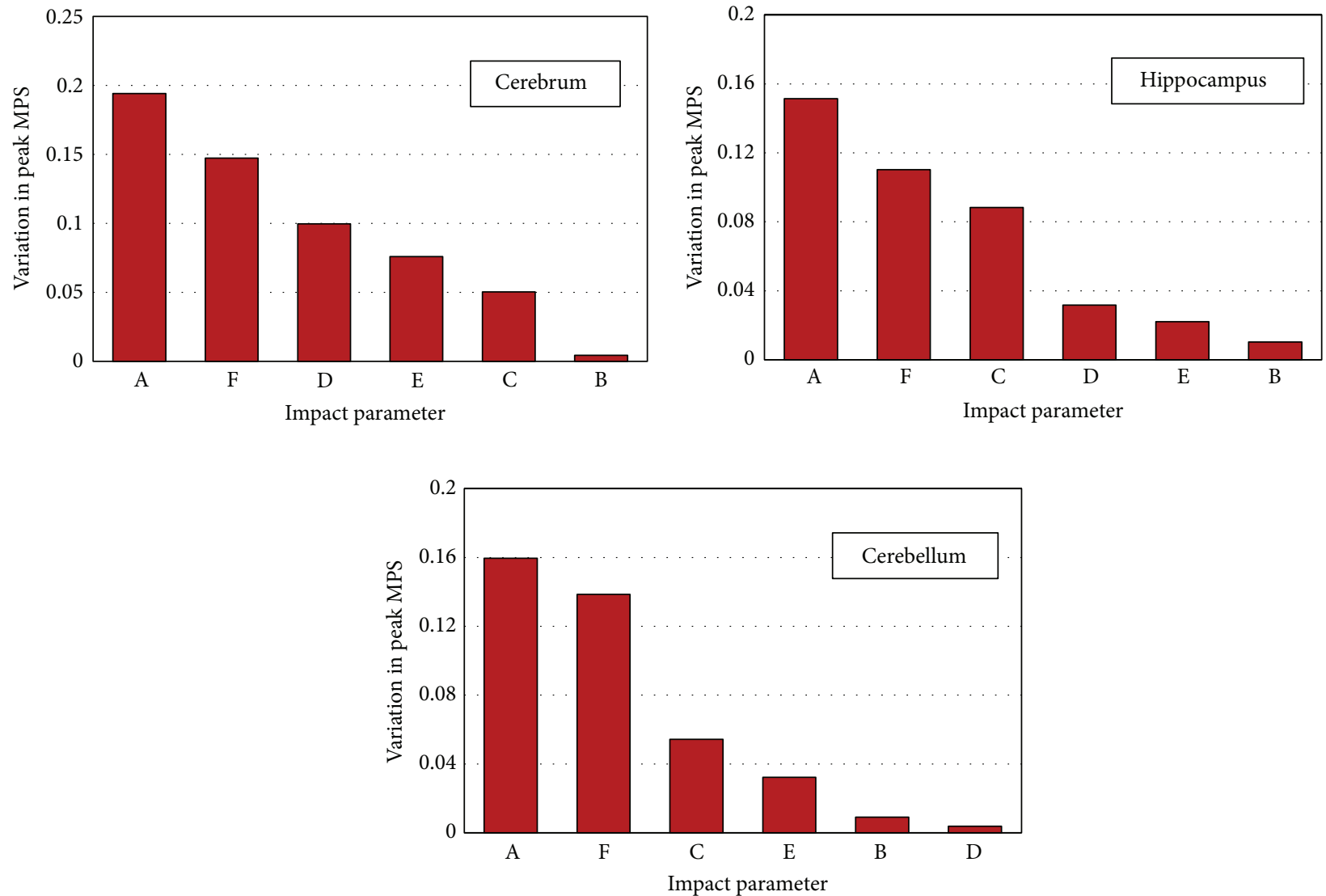

FIGURE 4: Pareto chart of region-specific biomechanical responses to external impact parameters. A: impact depth; B: impact velocity; C: impact position; D: impactor diameter; E: impactor material; F: impactor shape.

A larger $R$ indicates that the corresponding factor plays a more important role in the brain responses. The Pareto chart (Figure 4), based on the magnitude of range value $R$, has shown that impact depth and impactor shape were the two leading factors affecting biomechanical responses of the brain regardless of regions. For example, varying the impact depth from $1 \mathrm{~mm}$ to $2 \mathrm{~mm}$ produced an increase of peak MPS in the cerebrum of 0.1941 , in the hippocampus of 0.1514 , and in the cerebellum of 0.1595 . As a flat impactor was changed to a convex one, the peak MPS decreased by $0.1473,0.1102$, and 0.1386 in the cerebrum, hippocampus, and cerebellum, respectively. Moreover, the high strains induced by the flat 


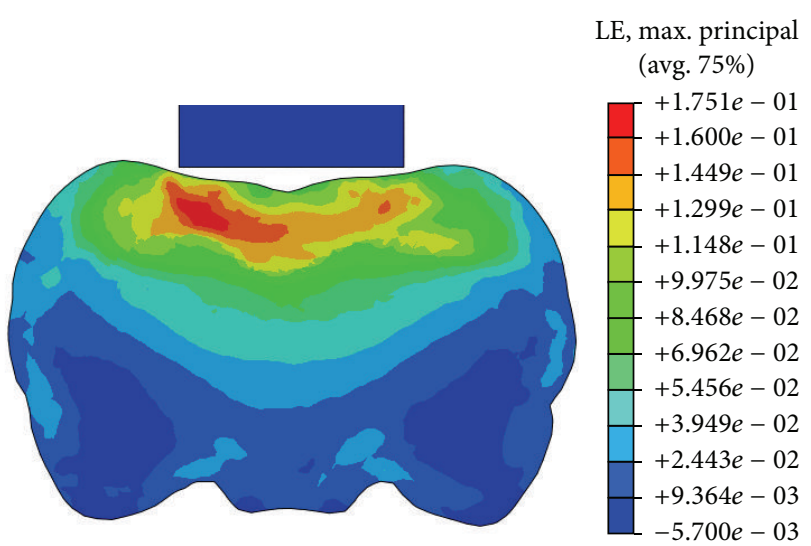

Case 1: flat impactor, central impact

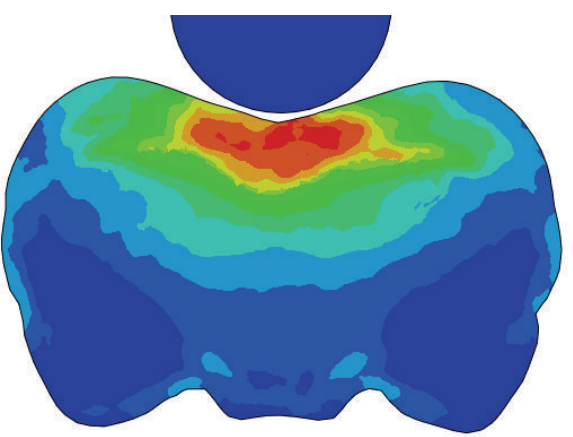

LE, max. principal (avg. 75\%)

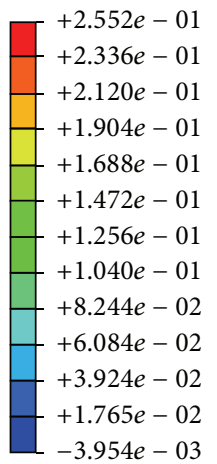

Case 7: convex impactor, central impact

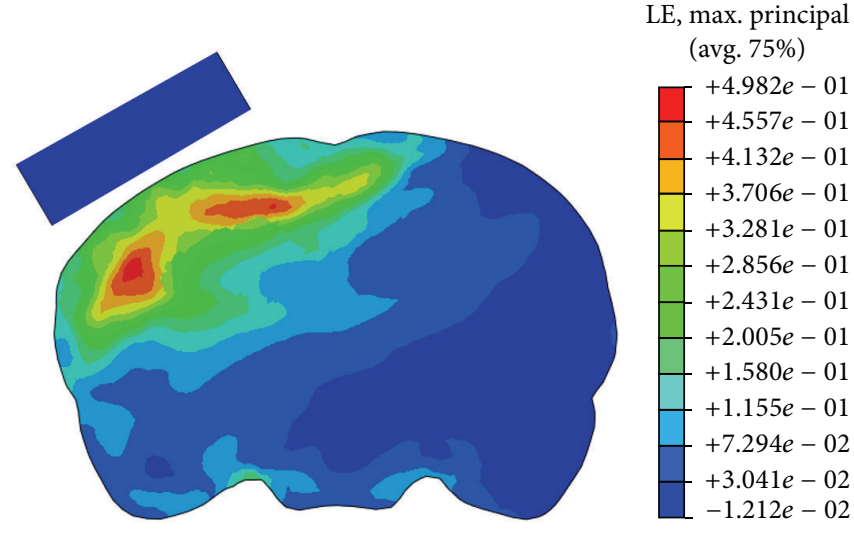

Case 8: flat impactor, lateral impact

FIGURE 5: Contour plots of maximum principal strain (MPS) on a coronal plane of the rat brain.

impactor were approximately parallel to the bottom rim of the impactor, while those induced by the convex one were concentrated along the axial line (Figure 5, Cases 1 and 7).

Following impact depth and impactor shape, impactor diameter ranked as the third most important factor for the calculated peak MPS in the cerebrum. However, impactor diameter had much less effect in the hippocampus and cerebellum. For example, the peak MPS in the cerebrum increased by 0.0997 when impactor diameter increased from $6 \mathrm{~mm}$ to $12 \mathrm{~mm}$, compared to a limited increase of 0.0318 and 0.0038 in the hippocampus and cerebellum, respectively.

Impact position seems to have a uniform effect on the biomechanical responses in all three brain regions. Changing the impact position from central to lateral, the peak MPS in the cerebrum, hippocampus, and cerebellum increased by $0.06 \pm 0.02$. A coronal view of the rat brain exhibited totally different strain patterns when the impact position was changed (Figure 5, Cases 1 and 8). It is observed that a lateral impact induced higher MPS to the ipsilateral side of the brain and the strain magnitude in most of the region of the contralateral side was almost zero. In contrast, a similar strain gradient was found on both sides of the brain for the central impact.
Only small variations were found due to variations in impact velocity and impactor material on the predicted peak MPS in all three brain regions. Since impact depth, impactor shape, impactor diameter, and impact position could affect the intracranial responses both qualitatively and quantitatively, they are most critical when designing appropriate $\mathrm{CHI}$ models.

\section{Discussion}

In this work, the intracranial responses to various impact parameters in the CHI model were systematically investigated using the 3D FE rat head model. The peak MPS was chosen as the response variable since previous investigations have demonstrated that regions with higher MPS correlated well with the brain injury severity including contusion volumes and the percentage of neuronal cell loss [18, 19]. Our model was first verified by previously published CCI injury data [13] and good agreement has been achieved (Figure 3). It is observed that the peak MPS predicted by the FE model was generally lower than the published results. This could be attributed to the difference in weight and size of the rat which we used. The anatomy and geometry of the brain used 


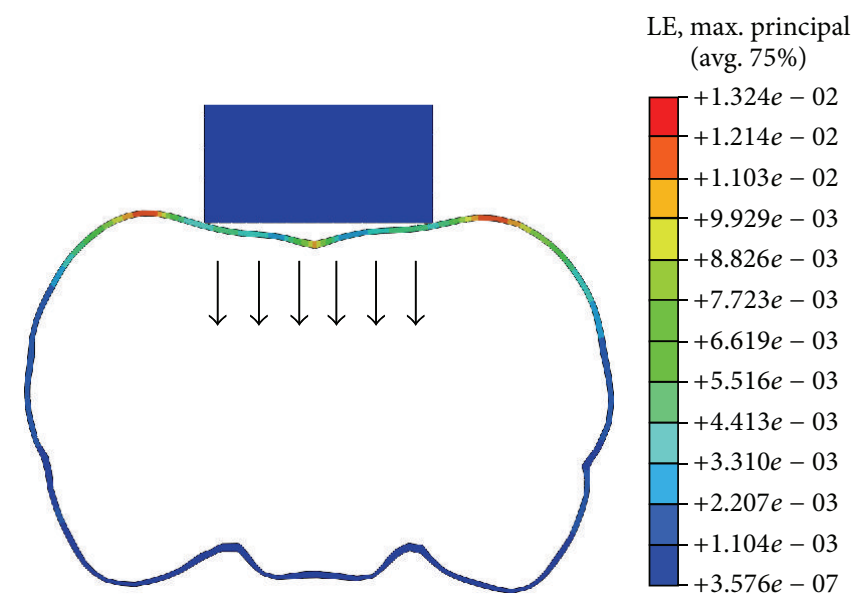

(a)

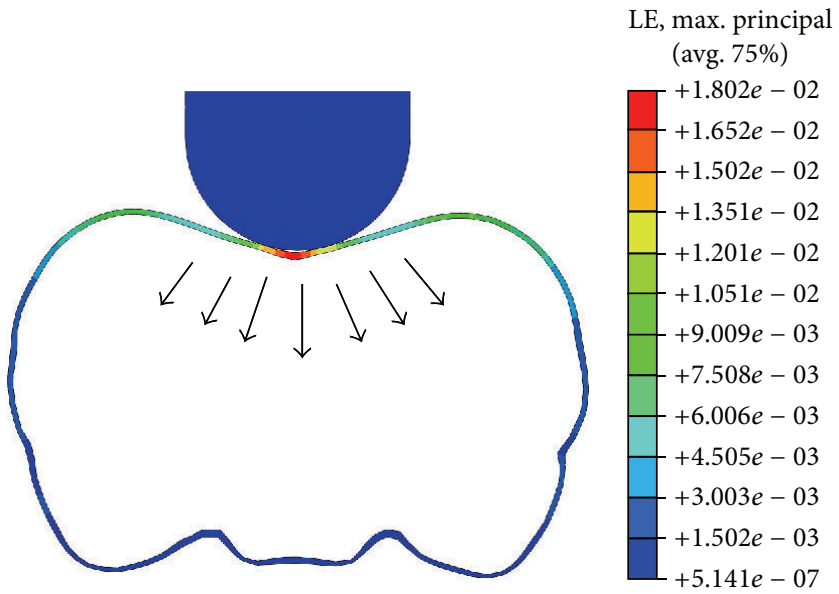

(b)

FIGURE 6: A coronal view of skull deformation for (a) flat impactor and (b) convex impactor.

in this work were taken from the rat weighing about $360 \mathrm{~g}$, which was larger than that of 250-300 $\mathrm{g}$ in [13]. A recent study has demonstrated that increasing the size of rat brain would decrease the magnitude of predicted strain under impact loading [20].

Following model verification, an orthogonal experimental design was then used to quantify the significance levels of six impact parameters on the brain response. Our results (Figure 4) have shown that the prescribed impact depth is the leading factor affecting intracranial MPS responses in CHI. A larger impact depth could result in more severe axonal damage as well as increased permeability of the blood-brain barrier in the rat brain $[7,12]$. This reinforced our finding that the intracranial peak MPS might serve as an index for the brain injury severity. On the contrary, the impact depth itself is not a reliable index based on observations that the impactinduced peak brain MPS could vary $103.2 \%$ in the cerebrum, $85.7 \%$ in the hippocampus, and $214.2 \%$ in the cerebellum under the same prescribed impact depth at $2 \mathrm{~mm}$.

Under the same boundary constrains, the convex impactor reduced the intracranial peak MPS compared to the flat one: that is, $K_{2}<K_{1}$ (column $\mathrm{F}$ in Table 3 ). However, it induced strain concentration within the brain (Figure 5, Cases 1 and 7), associated with the severity of TBI, and a stress concentration in the skull, leading to higher incidence of skull fracture (Figure 6). This is why flat impactors were commonly used in documented studies to induce the diffuse injury within the brain and to reduce the skull fracture $[3,5-7,9]$. In addition, the impactor diameter in the CHI tests is generally larger than $6 \mathrm{~mm}[3,6,7,9]$, whereas it is less than $5 \mathrm{~mm}$ in the CCI tests [11, 15]. An increase in impactor diameter could substantially increase tissue strains in the cerebrum, located directly beneath the impactor; however, the strains in the hippocampus and cerebellum seem to be not sensitive to this impact parameter.
Impact position is found to be crucial in determining the extent and location of tissue injury in rat brain. Our results (Table 3) show that lateral impact induced higher peak MPS in all three brain regions compared to the central impact. Moreover, the high strains induced by the lateral impact were mainly focused on the ipsilateral side of the brain, while those induced by the central impact were more diffusely located on both sides (Figure 5, Cases 1 and 8). This is consistent with the clinical observation that lateral impact inflicts primarily unilateral cortical damage, whereas central impact causes bilateral cortical alterations [3, 7]. Furthermore, the lateral impact is able to induce a larger relative movement between the brain and skull, which contributes to the subarachnoid hemorrhage (SAH), a common cerebrovascular event following $\mathrm{CHI}[5,6]$. The relative displacement at five marked locations (M1-M5) along the brain/skull interface was compared between central and lateral impacts (Figure 7). The maximum relative displacement was $0.43 \mathrm{~mm}$ at location $\mathrm{M} 1$ subjected to lateral impact and $0.20 \mathrm{~mm}$ at location $\mathrm{M} 2$ subjected to central impact. This indicates that $\mathrm{SAH}$ is more likely to occur on the ipsilateral side of the brain. However, the relative displacement induced by the lateral impact was minimal at location M2. This is due to the fact that this location is along the midline of the lateral impactor, which constrained the relative motion between the brain and skull. A similar observation existed at location M3, along the midline of the central impactor, which limited the relative skull/brain displacement. This implies that a properly designed impact position is able to guide SAH to target a specific region of interest.

The effect of impactor material on the intracranial responses is found to be very limited. This indicates that the brain injury severity might not be sensitive to the impactor materials. However, a softer impactor is commonly recommended for the CHI to avoid skull fracture $[3,9]$. To examine the significance of impactor materials on skull fracture using 

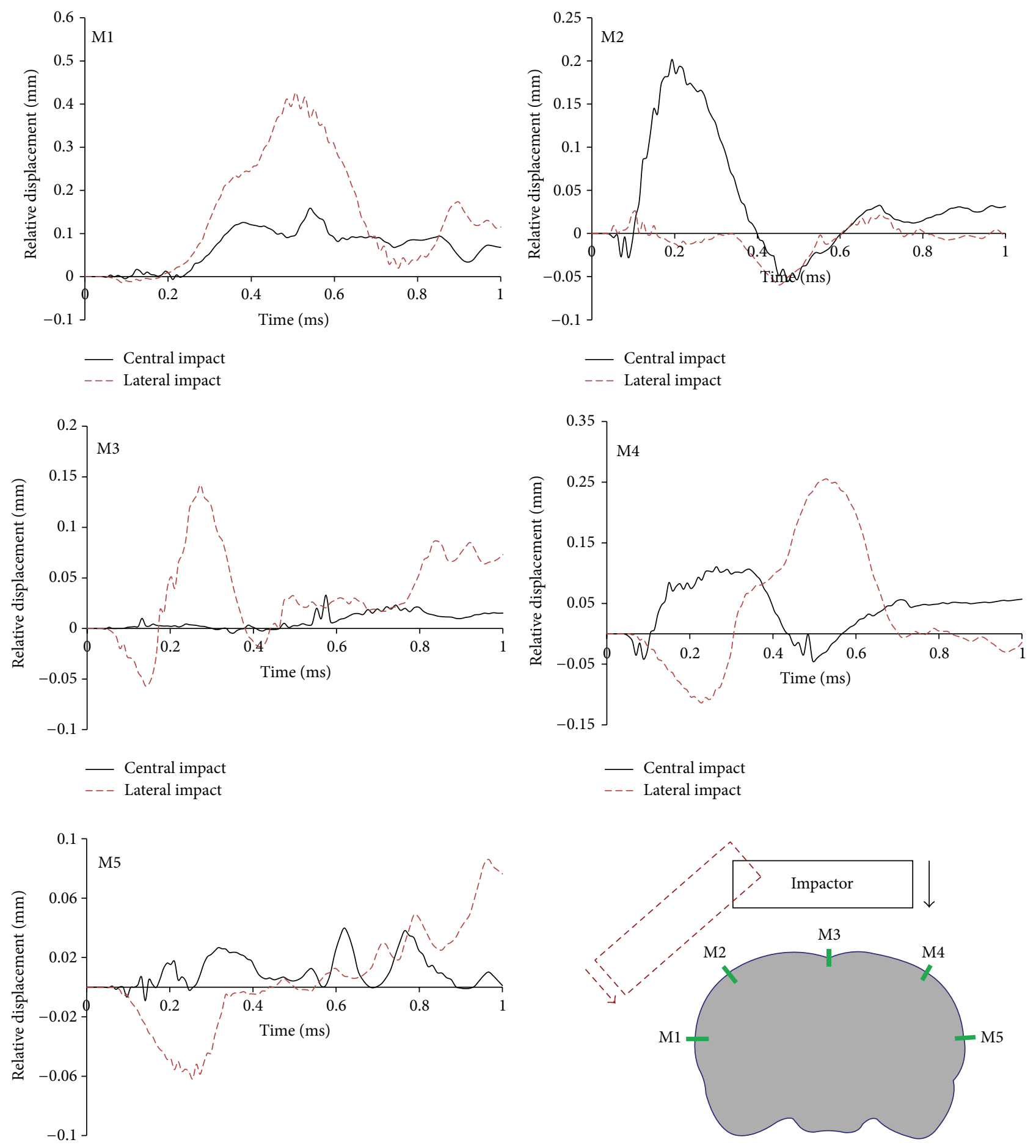

Central impact

- - - Lateral impact

FIGURE 7: Relative displacement at five mark locations (M1-M5) along the brain/skull interface. 
von Mises criteria, the peak von Mises stress in the rat skull was obtained for two impactor materials (e.g., steel and nylon). It is found that the peak stress decreased only by $1.87 \%$ when the impactor material changed from steel to nylon, indicating the almost same probability of skull fracture. Careful attention should be paid when the impactor is made of an extremely soft material such as rubber [4]. One more simulation was conducted using baseline data except the impactor materials. The rubber impactor-induced peak von Mises stress was $36.1 \%$ less than the steel one. It should be noted, though, that such reduction of skull stress is caused by the insufficiency of indentation depth. We measured a $1 \mathrm{~mm}$ shortening of the rubber impactor at the time of maximum indentation. This indicated that the actual indentation depth was $1 \mathrm{~mm}$ instead of the prescribed $2 \mathrm{~mm}$ impact depth, leaving the rat brain to be less injured. It is suggested to calibrate the prescribed impact depth or monitor the actual indentation depth when adopting a very soft impactor in the CHI tests.

It is interesting to find that variations in impact velocity induced only small variations on peak MPS in all three brain regions. On the other hand, a wide range of impact velocities are used in different laboratories with the goal to induce desired injury levels $[6,8]$. This inconsistency could be attributed to the "overshoot" phenomenon which widely exists in the pneumatically driven impact devices [21]. Overshoot is referred to as the maximum transient displacement of the impactor tip that exceeds the predefined impact depth. We have captured the trajectory of the impactor tip of a commercially available pneumatic device TBI-0310 Impactor (Precision Systems and Instrumentation) using a high-speed camera (Photron SA 1.1). It was observed that overshoot was positively correlated with the impactor velocity. For a predefined impact depth of $3 \mathrm{~mm}$, the device produced a $0.4 \mathrm{~mm}(13 \%)$ overshoot at an impact velocity of $2 \mathrm{~m} / \mathrm{s}$, while the overshoot was substantially increased to $1.5 \mathrm{~mm}(50 \%)$ at $5 \mathrm{~m} / \mathrm{s}$. This overshoot phenomenon is not considered in our computational models, which might underestimate the role of impact velocity. In addition, the rat skull was modeled as a uniform thin layer, rather than geometrical details such as varied thickness.

The brain tissue was simplified as an isotropic homogeneous material, rather than a site-dependent white and gray matter combination including a dense network of blood vessels, cellular structure, or differing cell types such as glial cells which are highly responsive to brain injury [22-24]. The exclusion of these elements might alter the magnitude of brain dynamics. Moreover, the rat brain was meshed using tetrahedral elements. Although this element type makes meshing complex geometries easier, it tends to exhibit a stiffer response when compared to quadrilateral or hexahedral meshes because of shear/volumetric locking. Despite these simplifications, the present work demonstrated the significance level of six input parameters in terms of the brain MPS, which may have significant clinical implications for brain injury. This work can be used to provide a fundamental understanding of the impact of CHI designs on the brain and to better design a site- and severity-specific rodent $\mathrm{CHI}$ model.

\section{Disclosure}

This work is submitted as an original paper.

\section{Conflict of Interests}

The authors declare that there is no conflict of interests regarding the publication of this paper.

\section{Acknowledgments}

The authors acknowledge Mr. Aravind Sundaramurthy for his help in segmenting different rat brain regions using Mimics. The authors also thank Dr. Michael Boska from University of Nebraska Medical Center for proving MRI rat brain data and Mr. Gang Zhao for operating the pneumatically driven impact device.

\section{References}

[1] B. W. de Witt, K. M. Ehrenberg, R. L. McAloon et al., "Abbreviated environmental enrichment enhances neurobehavioral recovery comparably to continuous exposure after traumatic brain injury," Neurorehabilitation and Neural Repair, vol. 25, no. 4, pp. 343-350, 2011.

[2] E. R. Peskind, D. Brody, I. Cernak, A. McKee, and R. L. Ruff, "Military- and sports-related mild traumatic brain injury: clinical presentation, management, and long-term consequences," Journal of Clinical Psychiatry, vol. 74, no. 2, pp. 180-188, 2013.

[3] H. L. Laurer, F. M. Bareyre, V. M. Y. C. Lee et al., "Mild head injury increasing the brain's vulnerability to a second concussive impact," Journal of Neurosurgery, vol. 95, no. 5, pp. 859-870, 2001.

[4] Y. Shitaka, H. T. Tran, R. E. Bennett et al., "Repetitive closedskull traumatic brain injury in mice causes persistent multifocal axonal injury and microglial reactivity," Journal of Neuropathology and Experimental Neurology, vol. 70, no. 7, pp. 551-567, 2011.

[5] D. T. Laskowitz, S. E. McKenna, P. Song et al., "COG1410, a novel apolipoprotein E-based peptide, improves functional recovery in a murine model of traumatic brain injury," Journal of Neurotrauma, vol. 24, no. 7, pp. 1093-1107, 2007.

[6] K. Maruichi, S. Kuroda, Y. Chiba et al., "Graded model of diffuse axonal injury for studying head injury-induced cognitive dysfunction in rats," Neuropathology, vol. 29, no. 2, pp. 132-139, 2009.

[7] I. Cernak, R. Vink, D. N. Zapple et al., "The pathobiology of moderate diffuse traumatic brain injury as identified using a new experimental model of injury in rats," Neurobiology of Disease, vol. 17, no. 1, pp. 29-43, 2004.

[8] Z. Ren, J. J. Iliff, L. Yang et al., “'Hit \& Run’ model of closed-skull traumatic brain injury (TBI) reveals complex patterns of posttraumatic AQP4 dysregulation," Journal of Cerebral Blood Flow \& Metabolism, vol. 33, no. 6, pp. 834-845, 2013.

[9] L. Longhi, K. E. Saatman, S. Fujimoto et al., “Temporal window of vulnerability to repetitive experimental concussive brain injury," Neurosurgery, vol. 56, no. 2, pp. 364-373, 2005.

[10] G. G. Gurkoff, J. D. Gahan, R. T. Ghiasvand et al., "Evaluation of metric, topological, and temporal ordering memory tasks after lateral fluid percussion injury," Journal of Neurotrauma, vol. 30, no. 4, pp. 292-300, 2013. 
[11] C. M. Atkins, M. L. Cepero, Y. Kang, D. J. Liebl, and W. D. Dietrich, "Effects of early rolipram treatment on histopathological outcome after controlled cortical impact injury in mice," Neuroscience Letters, vol. 532, no. 1, pp. 1-6, 2013.

[12] A. Levchakov, E. Linder-Ganz, R. Raghupathi, S. S. Margulies, and A. Gefen, "Computational studies of strain exposures in neonate and mature rat brains during closed head impact," Journal of Neurotrauma, vol. 23, no. 10, pp. 1570-1580, 2006.

[13] H. J. Mao, X. Jin, L. .Y. Zhang et al., "Finite element analysis of controlled cortical impact-induced cell loss," Journal of Neurotrauma, vol. 27, no. 5, pp. 877-888, 2010.

[14] A. Gefen, N. Gefen, Q. Zhu, R. Raghupathi, and S. S. Margulies, "Age-dependent changes in material properties of the brain and braincase of the rat," Journal of Neurotrauma, vol. 20, no. 11, pp. 1163-1177, 2003.

[15] H. Mao, L. Zhang, K. H. Yang, and A. I. King, "Application of a finite element model of the brain to study traumatic brain injury mechanisms in the rat," Stapp Car Crash Journal, vol. 50, pp. 583-600, 2006.

[16] J. D. Finan, B. S. Elkin, E. M. Pearson, I. L. Kalbian, and B. Morrison, "Viscoelastic properties of the rat brain in the sagittal plane: effects of anatomical structure and age," Annals of Biomedical Engineering, vol. 40, no. 1, pp. 70-78, 2012, Erratum in Annals of Biomedical Engineering, vol. 40, no. 4, pp. 982-985, 2012.

[17] H. Mao, C. Wagner, F. Guan, Y. N. Yeni, and K. H. Yang, "Material properties of adult rat skull," Journal of Mechanics in Medicine and Biology, vol. 11, no. 5, pp. 1199-1212, 2011.

[18] H. Mao and K. H. Yang, "Investigation of brain contusion mechanism and threshold by combining finite element analysis with in vivo histology data," International Journal for Numerical Methods in Biomedical Engineering, vol. 27, no. 3, pp. 357-366, 2011.

[19] M. B. Elliott, J. J. Jallo, and R. F. Tuma, "An investigation of cerebral edema and injury volume assessments for controlled cortical impact injury," Journal of Neuroscience Methods, vol. 168, no. 2, pp. 320-324, 2008.

[20] H. J. Mao, K. H. Yang, A. I. King, and K. Yang, "Computational neurotrauma-design, simulation, and analysis of controlled cortical impact model," Biomechanics and Modeling in Mechanobiology, vol. 9, no. 6, pp. 763-772, 2010.

[21] D. L. Brody, C. Mac Donald, C. C. Kessens et al., "Electromagnetic controlled cortical impact device for precise, graded experimental traumatic brain injury," Journal of Neurotrauma, vol. 24, no. 4, pp. 657-673, 2007.

[22] A. Nimmerjahn, F. Kirchhoff, and F. Helmchen, "Resting microglial cells are highly dynamic surveillants of brain parenchyma in vivo," Science, vol. 308, no. 5726, pp. 1314-1318, 2005.

[23] M. L. Kelso and H. E. Gendelman, "Bridge between neuroimmunity and traumatic brain injury," Current Pharmaceutical Design, vol. 20, no. 26, pp. 4284-4298, 2014.

[24] Y. Hua, S. Lin, and L. Gu, "Relevance of blood vessel networks in blast-induced traumatic brain injury," Computational and Mathematical Methods in Medicine, In press. 

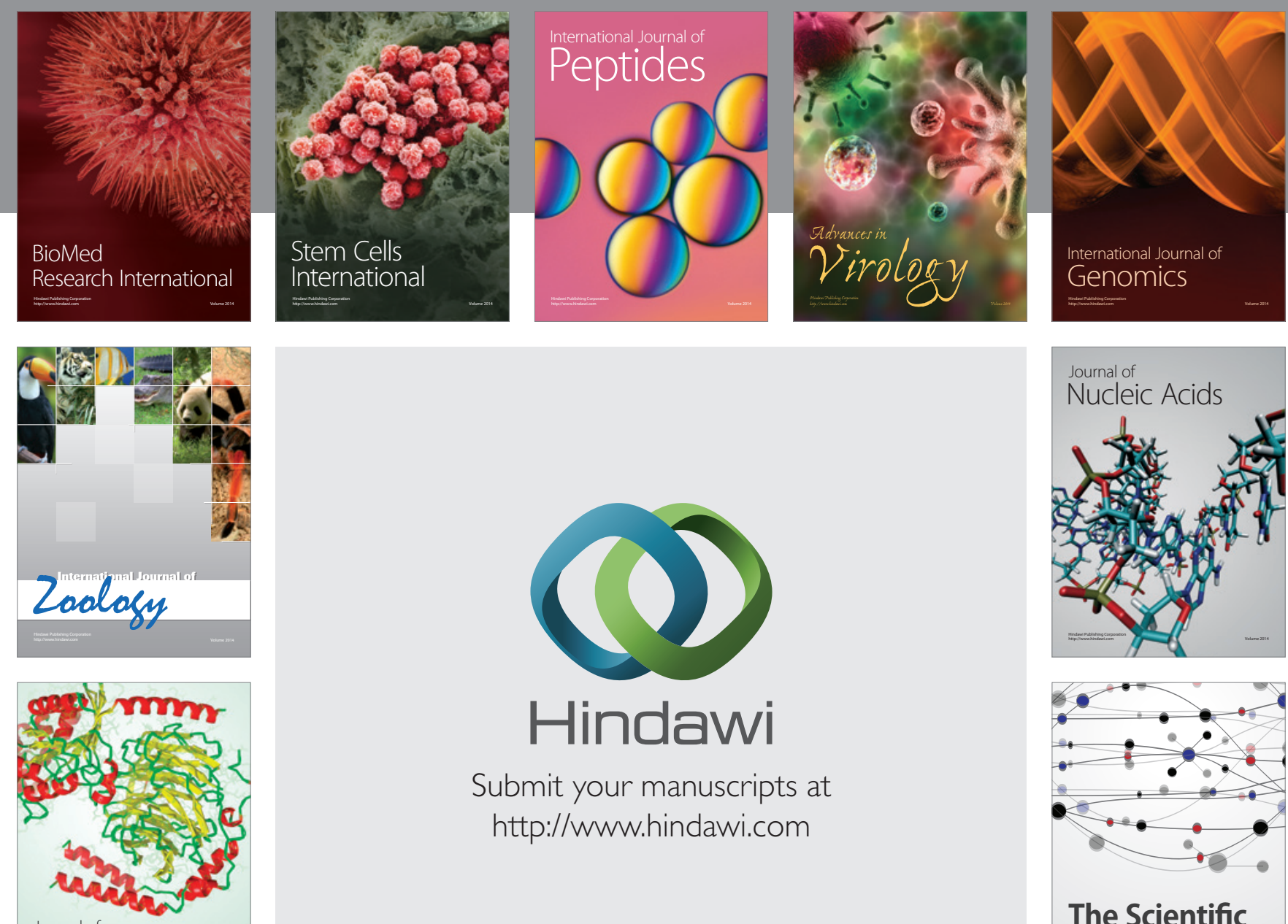

Submit your manuscripts at

http://www.hindawi.com

Journal of
Signal Transduction
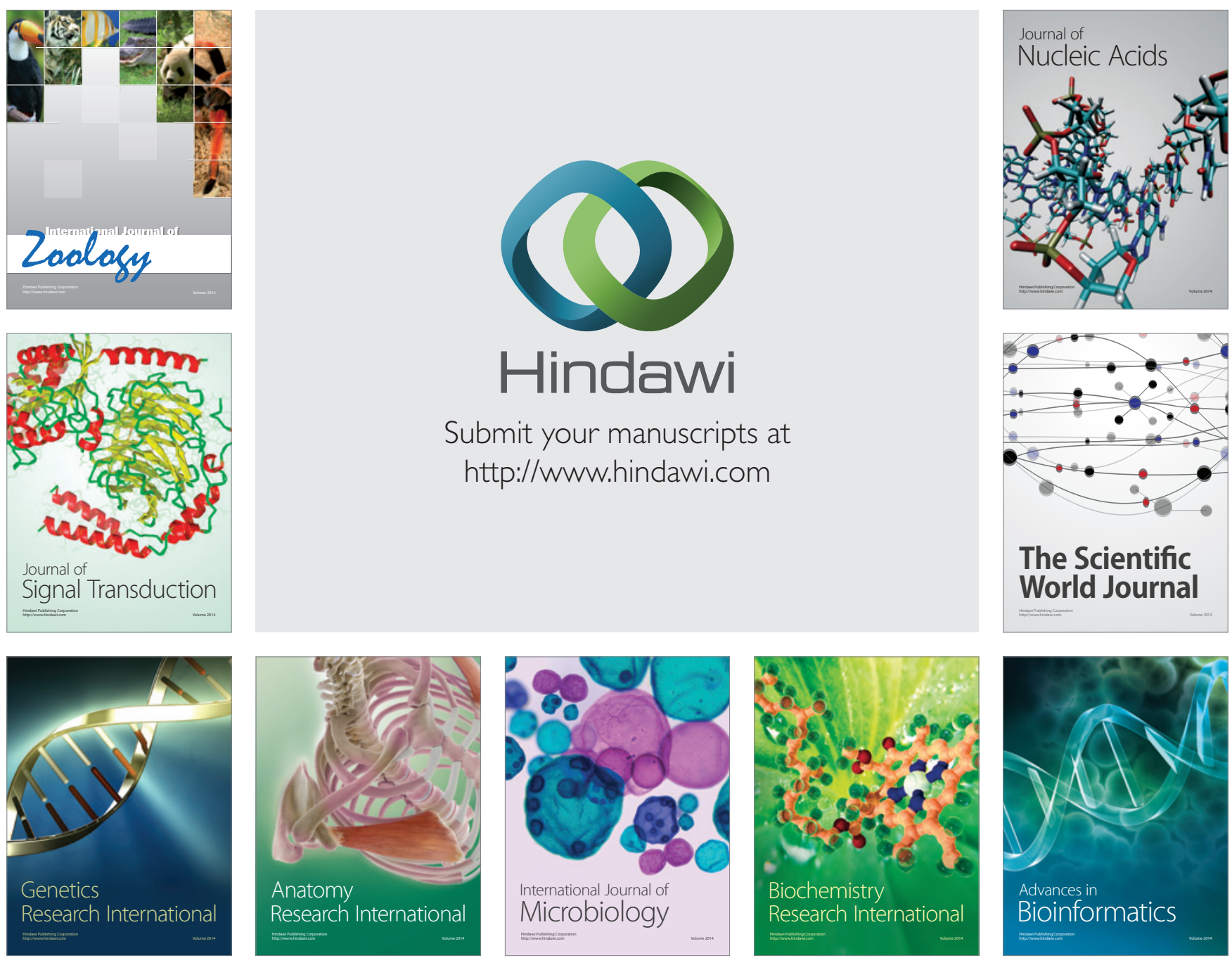

The Scientific World Journal
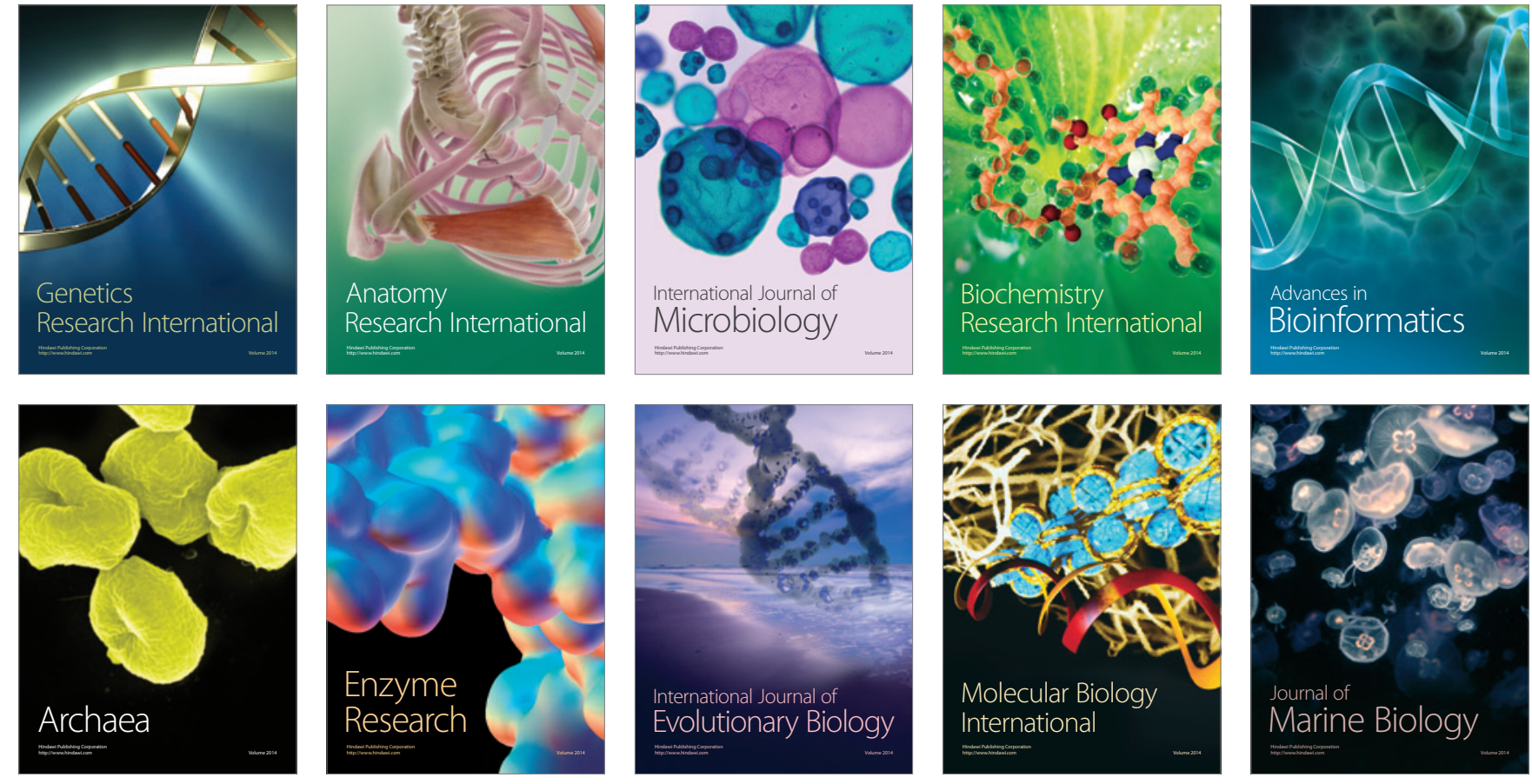